\title{
RHIC FY pp Run RHIC and AGS polarization analysis
}

\author{
H. Huang, P. Adams
}

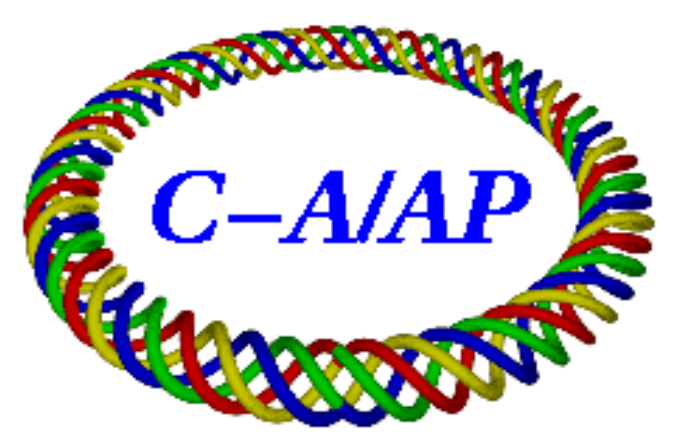

\section{Collider-AcceleratorDepartment Brookhaven National Laboratory Upton, NY 11973}

\author{
U.S. Department of Energy \\ Office of Science, Office of Nuclear Physics
}

Notice: This document has been authorized by employees of Brookhaven Science Associates, LLC under Contract No. DE-SC0012704 with the U.S. Department of Energy. The United States Government retains a nonexclusive, paid-up, irrevocable, world-wide license to publish or reproduce the published form of this document, or allow others to do so, for United States Government purposes. 


\title{
RHIC FY15 pp Run RHIC and AGS Polarization Analysis
}

\author{
H. Huang, P. Adams \\ (Dated: February 20, 2016)
}

\begin{abstract}
The polarization information is important for the spin physics program in Relativistic Heavy Ion Collider (RHIC). There are discrepancies between AGS and RHIC polarization measurements. First, the face value of AGS polarization is higher than RHIC ones in general. Second, the measured polarization profile (described by the profile ratio $R$ ) is stronger in AGS than in RHIC. This note analyzes the polarization data from FY15 pp run period. The results show that the differences between AGS and RHIC polarization measurements are reasonable, but the $R$ value difference is puzzling. The difference between blue and yellow ring is worth of spin simulation to explain.
\end{abstract}

\section{INTRODUCTION}

In FY15 polarization proton(pp) run, there were several differences from past runs. First, the source upgrade made it possible to double Booster input intensity which in turn allowed heavier Booster transverse scraping to reach small emittance. Second, the electron lens in RHIC allowed higher bunch intensity to be injected in RHIC. There are also changes related to the polarimeters. To accommodate the e-lens, the lattice is modified such that the beta functions at polarimeters are larger. This change prohibits the use of blue vertical and yellow horizontal targets at RHIC injection, because the significant beam loss was generated by beam hitting target frame. As the result, only one polarization measurement was taken for each ring at injection. Second, the small fins are added to 4 out of 6 targets to reduce the target heating [1]. This also reduced the available target portion for beam to hit the target as the fin blocks the flying path for the Carbon nuclei generated at the edges of a target. But overall target lifetime is longer, probably benefited from the fins and larger beam sizes.

There are three periods in FY15 pp run: 1st longitudinal polarization run, 18646-18741; transverse polarization run, 18749-18857; 2nd longitudinal polarization run, 18868-18953. The bunch intensity was ramped up during the first period and continued to be ramped up during the second period. The source polarization, AGS extraction polarization and Blue ring injection bunch intensity is plotted in Fig. 1.

There are apparent difference in average polarization among three different RHIC periods. It can be seen from the jet polarization measurements shown in Fig. 2. The yellow polarization is going down over the three periods modestly, which is associated with higher intensity. The blue polarization drop is quite significant, which indicates blue setting changes affected polarization in addition to the lower polarization associated with higher intensity.

Here are a few phenomena observed:

1. There is a big difference ( $10 \%$ ) between AGS and RHIC injection polarization measurements [2]. It also seems that the transfer efficiency was worse later in the run when bunch intensity increased. Since AGS ones are fixed target

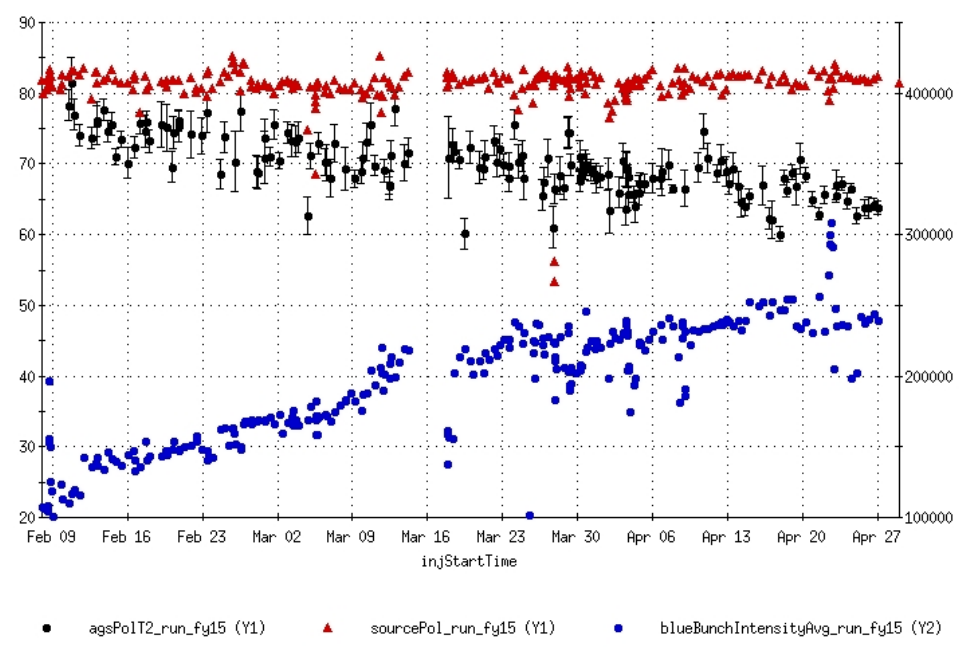

FIG. 1: Source polarization during RHIC fills (red triangles, left axis), AGS extraction polarization for RHIC fills(black dots, left axis) and blue bunch intensity of RHIC fills (blue dots, right axis). Blue intensity was stable around $2.3 \mathrm{E} 11$ at later part of run. The transverse period started on March 6. The second longitudinal period started on April 4 and stopped on April 27. 

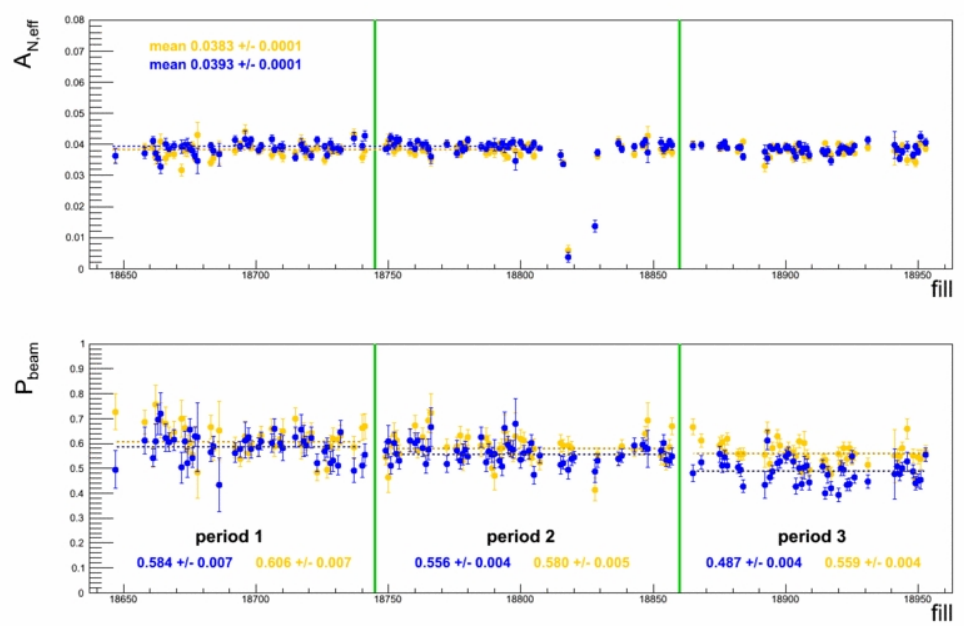

FIG. 2: The jet polarization in both rings for the three pp run periods. The plot is taken from Ref. [3]. The top plot is the analyzing power and the bottom plot is the beam polarization measured by polarized hydrogen jet.

measurement and RHIC ones are sweep measurements, the face values need to be converted for fair comparison.

2. The average polarization levels of three periods are different. There are RHIC setting differences in the three periods and intensity also varied in the three periods. From the jet polarization measurement, it is clear that the blue polarization is worse than yellow. There is contribution from RHIC side to the lower polarization. Can we quantify the polarization losses in the AGS, on the RHIC ramp and at RHIC store?

3. The $R$ value reported in RHIC is smaller than in AGS. Specifically, they are in the order of $0.05-0.07$ at injection and the beginning of store, but they are in the order of 0.11 at AGS extraction(see discussion in Section VI). Why they are different and what could be the possible reasons?

\section{ANALYSIS METHOD}

First, the RHIC injection and AGS polarization in the three periods are compared. The peak and average polarization should be converted for comparison. One easy way is to convert AGS fixed target measurement into sweep measurement results, based on $R$ values. In addition, the RHIC injection and AGS polarization ratio for individual fill is also compared and analyzed as function of bunch intensity. This analysis provides information on the AGS to RHIC polarization transfer efficiency and possible intensity dependence if any.

Second, we can analyze the polarization decay over the three different periods to see if RHIC polarization decay is different in the three periods. The known differences of the three periods are rotator settings, e-lens (it was on since middle of second period) and intensity. Similar analysis is available from polarimeter group's website. The difference here is that the polarization measurements were separated for horizontal and vertical targets, so the $R$ values for the two planes are obtained.

Thirdly, the polarization with zero emittance $P_{0}$ can be derived based on the measured $R$ values in RHIC to see if it is consistent with $R$ values from AGS.

The polarization profile and beam profile ratio $R$ is defined as [4]

$$
R=\left(\frac{\sigma_{b}}{\sigma_{p}}\right)^{2}
$$

For beam with depolarizing schemes in both $x$ and $y$ planes, there are two different $R$ values for the two planes. They can be different in general, but the observation is that both are about the same in AGS and RHIC.

\section{RHIC AVERAGE POLARIZATION}

The polarization measurements in RHIC were grouped by vertical and horizontal targets separately first. So the polarization profiles of both directions can be derived. The polarization data were taken from polarimeter group website which was analyzed in October, 2015 [3]. The store measurements were grouped as 0, 3, 6, 8 hours measurements. 
The time is determined from the "lumi" event. For some fills, there were more measurements beyond 8 hours. They are lumped into one category, 10 hours. There were not many of these runs so their impact on the fitting slope is not significant. It should be pointed out that the data can be analyzed for individual fills but the plots are less clear. The analysis was also carried out for individual fills and it is confirmed that the fitted polarization at beginning of store and slope of polarization loss are very close for the two methods.

For longitudinal running period, there were measurements before rotator ramp and they were labeled as before rotator ramp. Currently, the jet normalization factors obtained at store is applied to injection polarization. It is a small factor: about 1 in blue and around 1.05 for yellow. With this normalization, there is polarization loss on the energy ramp part. There is also polarization loss on the rotator ramp, as shown in RHIC retreat polarimeter presentation [2]. A python program was used to select data from logview data base. Polarization values above $95 \%$ and below $30 \%$ were not included in the data analysis as they were viewed as nonphysical.

\section{POLARIMETER OPERATION}

Polarization usually was measured in each ring once at injection. The run15 optical lattice has been changed to accommodate the introduction of e-lens in IP10. As the result, the beta function at polarimeter locations are significantly changed from the past. At injection, the vertical beta function is in the order of $55 \mathrm{~m}$ at blue 1 and blue 2 polarimeters, a increase of $80 \%$ from the past. Similarly, the horizontal beta function at yellow 1 and yellow 2 polarimeters is in the order of $65 \mathrm{~m}$ which is more than doubled from the past. The blue vertical targets and yellow horizontal targets caused significant beam loss and beam blow up, as beam tails hit the target frames (the target frame width is $2.5 \mathrm{~cm}$, which was not designed for such a large beam). Throughout the run, only very few polarization measurements were done with these two sets of targets. Since polarization profile information requires to average through many measurements, we only have polarization profile information for one plane in each ring: yellow horizontal and blue vertical.

The beam size is much smaller at store so the large beta function at store is not a problem. As a matter of fact, the larger beta function means the beam is distributed to larger portion of a target, which probably extended target lifetime. For longitudinal polarization periods, rotator ramp is needed. There are polarization measurements before and after rotator ramp. The one before is labeled as before rotator ramp. The one after is labeled as 0th hour measurement for the physics store. The polarization data were sorted out from database by their energy (for injection), by the time from ev-lumi (for store measurement). For store measurements, each time contains measurements done at given hour with +-0.5 hours window. For some stores, the store length is longer than 8 hours. All measurements done after 8.5 hours are lumped into 10th hour measurement. There are only a few those measurements, and they do not affect the fitting by much.

With polarization profile, the polarization of the whole beam and the polarization at beam center are different. Polarization can be measured by either fixed target measurement at beam center or a sweep measurement in which the target sweeps through beam with constant speed. The fixed target measurement gives peak polarization, $P_{p e a k}$. The sweep polarization gives the average polarization of the whole beam, $P_{\text {ave }}$.

\section{POLARIZATION DECAY AND PROFILES}

Fig. 3 showed the average polarization for blue ring at injection, at store before rotator ramp and through the store. The store polarization measurements is fitted linearly for the three periods and for them combined. First of all, the agreement for initial polarization and slope between vertical and horizontal targets are within error bars. Second, the overall polarization level is getting lower as intensity was ramped up over the three periods. The slope of last period (long.II) is stronger. Since yellow polarization (see below) did not show similar behavior, this can not be explained by higher intensity. The effect should be from blue optics. The emittance reported by IPM at RHIC injection did not show increase with higher intensity in last two periods. On the other hand, the AGS IPM emittance is larger for higher bunch intensity. Even with the modest increase of emittance, the two snakes should be able to overcome stronger resonance strength. The difference behavior of blue polarization in the long.II period is worth of study to find out the causes. The known differences are e-lens setting and subtle difference in optics.

Fig. 4 showed the average polarization for yellow ring at injection, at store before rotator ramp and through the store. Again, the agreement between vertical and horizontal targets is within error bars. Even though the polarization levels of the three periods are different, the slopes are similar in the three periods and they agreed within error bars. This is different from blue ring, which suggests the polarization loss in yellow at store is not correlated with higher intensity and possible larger emittance (last two periods), nor rotator settings. This is encouraging since the polarization loss rate can be independent of bunch intensity with proper settings. 

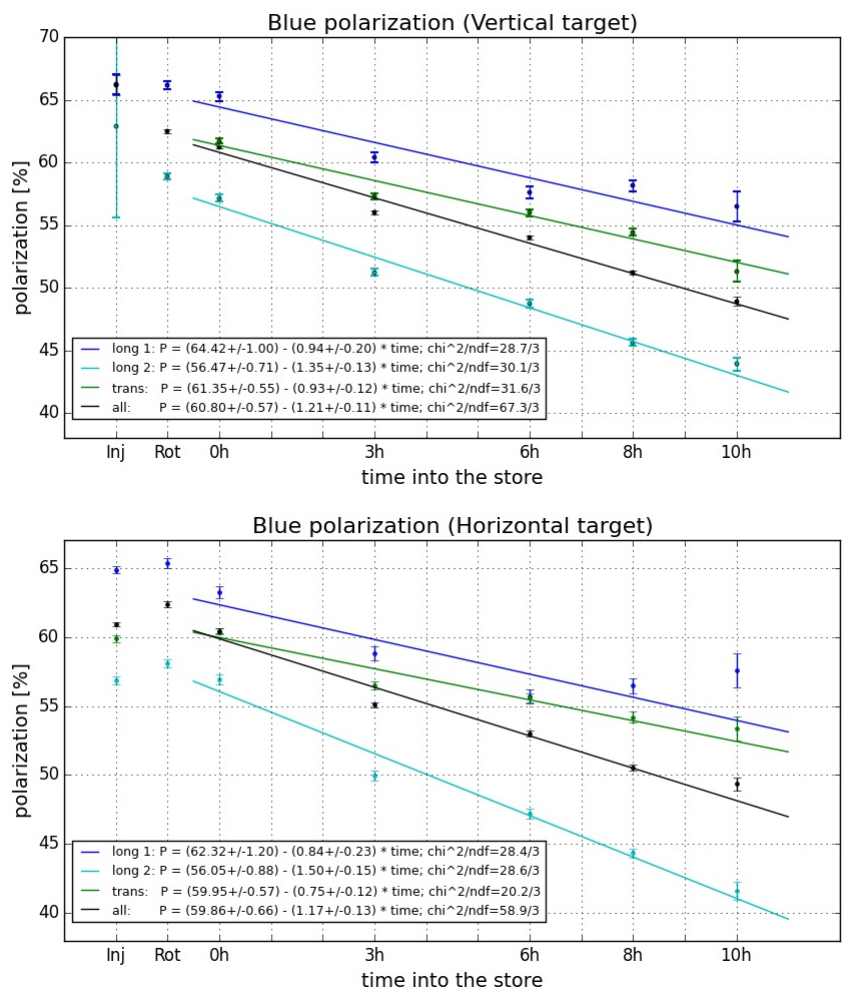

FIG. 3: Blue polarization from vertical targets (top) and horizontal targets (bottom). The injection measurements with vertical targets should be ignored as there were only very few measurements.
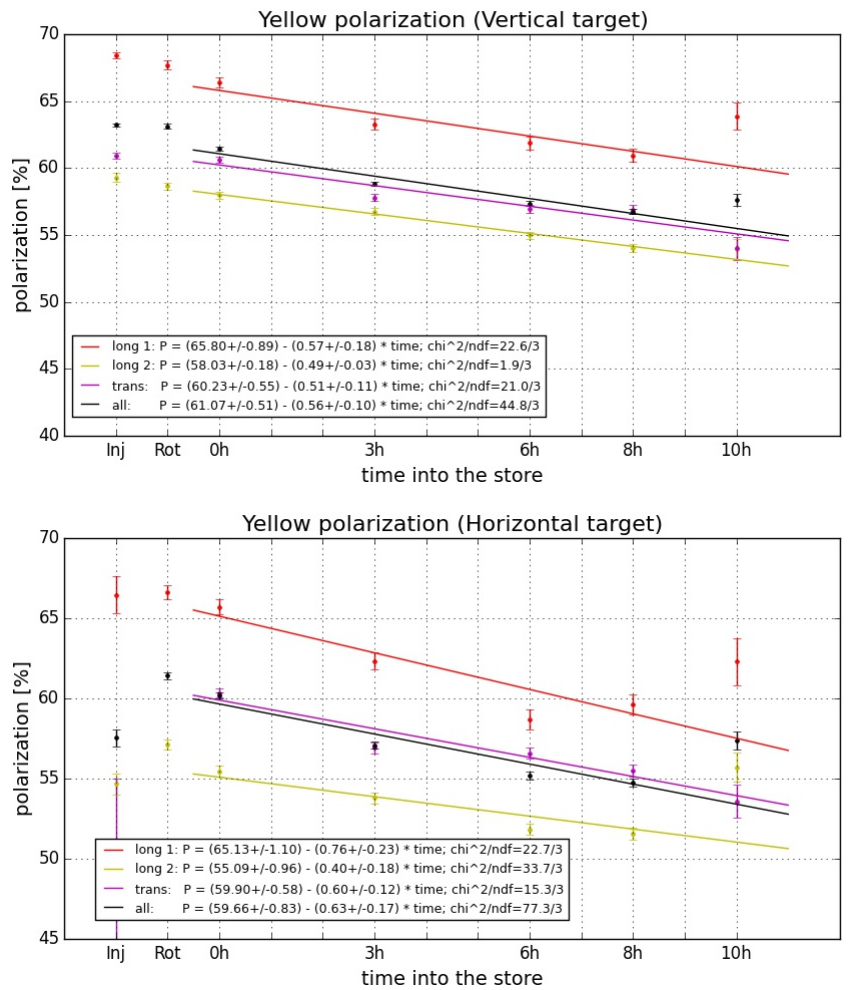

FIG. 4: Yellow polarization from vertical targets (top) and horizontal targets (bottom). The injection measurements with horizontal targets should be ignored as there were only very few measurements. 

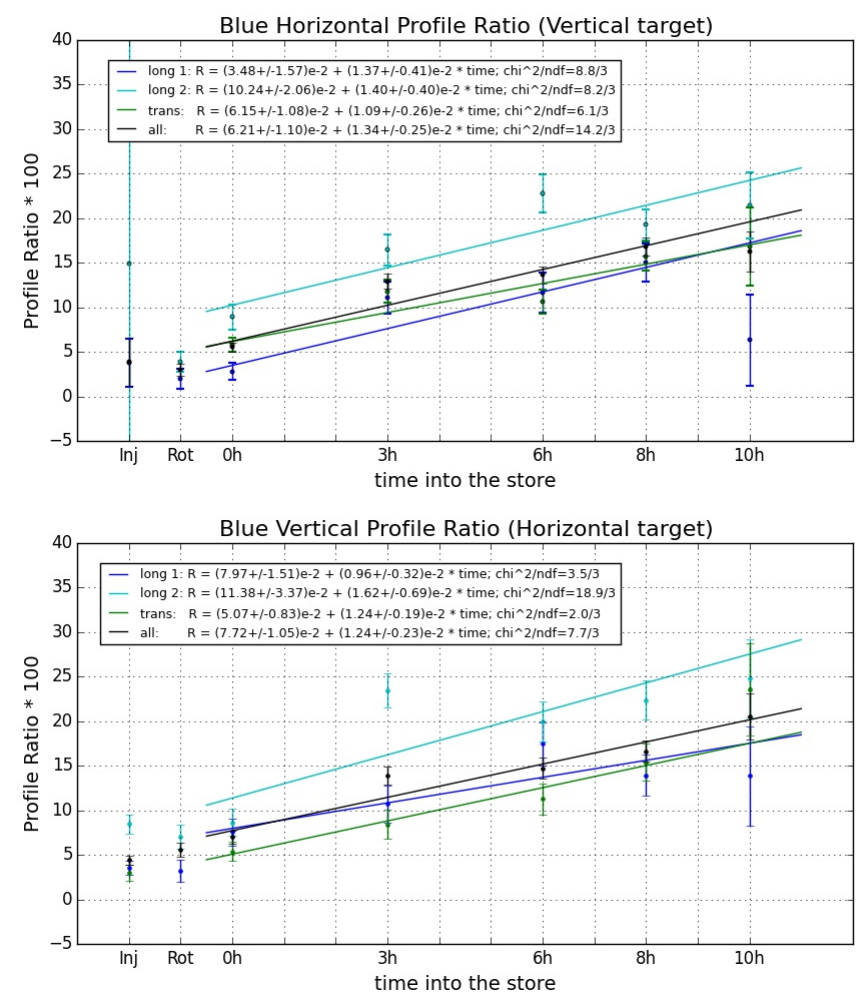

FIG. 5: Blue profile ratio from vertical targets (top) and horizontal targets (bottom).

Similar to polarization values, the polarization profiles as function of store time can also be derived from the averaged polarization profiles. Fig.5 shows the polarization profile ratios for blue ring. As the error bars are much larger compared to the polarization values, the chi square is improved. Overall trend for $\mathrm{R}$ value is to get larger over time in store, indicating polarization losses are associated with betatron amplitude (steeper polarization profiles). The initial values of $R$ is different for the three periods (consisting with the fact that the initial store polarization values are different, too), but the slopes are consistent within error bars. This is not expected. The larger slope of polarization decay in the 3rd period would lead to steeper slope in the $R$ value for the 3 rd period. Due to the large error bars in $R$ values, we could not distinguish the three periods for the $R$ values.

Similarly, the polarization profile ratios for yellow ring is shown in Fig. 6. The slope is in general smaller than in blue ring, as polarization decay is also smaller as shown in Fig. 4. The slopes in general are similar for all three periods. Although the error bars for individual periods are large, the error bars for all three periods combined are smaller. It shows that the slope of yellow ring is much better than that for blue ring, consistent with better polarization for yellow: similar input polarization from AGS, but different polarization loss rate at store.

Since bunch intensity was ramped up during transverse polarization period, one can split this period into two parts: intensity ramping up part and intensity stable part. Such analysis was done. The results show that the polarization decay rate for the two parts are in agreement within error bars. The initial polarization at the beginning of store is higher for the intensity ramping up part. This confirms that the major cause of polarization difference is the polarization out of AGS, and the AGS polarization is correlated with intensity (or emittance).

The comparison between blue and yellow(Figs. 3-6 and Table I shows that the polarization at the beginning of store (fitted polarization at 0th hour) is almost the same although a $3 \%$ difference is expected from the spin mismatch difference. But the slope is much stronger in blue, which resulted lower overall polarization.

\section{AVERAGE AND ZERO EMITTANCE POLARIZATION}

With both $P_{\text {ave }}$ and $R$ values measured, one can derive zero emittance polarization $P_{0}$ throughout the store. In the presence of two full snakes, the polarization loss in RHIC can be due to snake resonances associated with strong intrinsic resonances. This type of polarization loss depends on the betatron amplitude and should result in polarization profile. In this scenario, the zero emittance polarization $P_{0}$ should stay constant through accelerator chain (except 

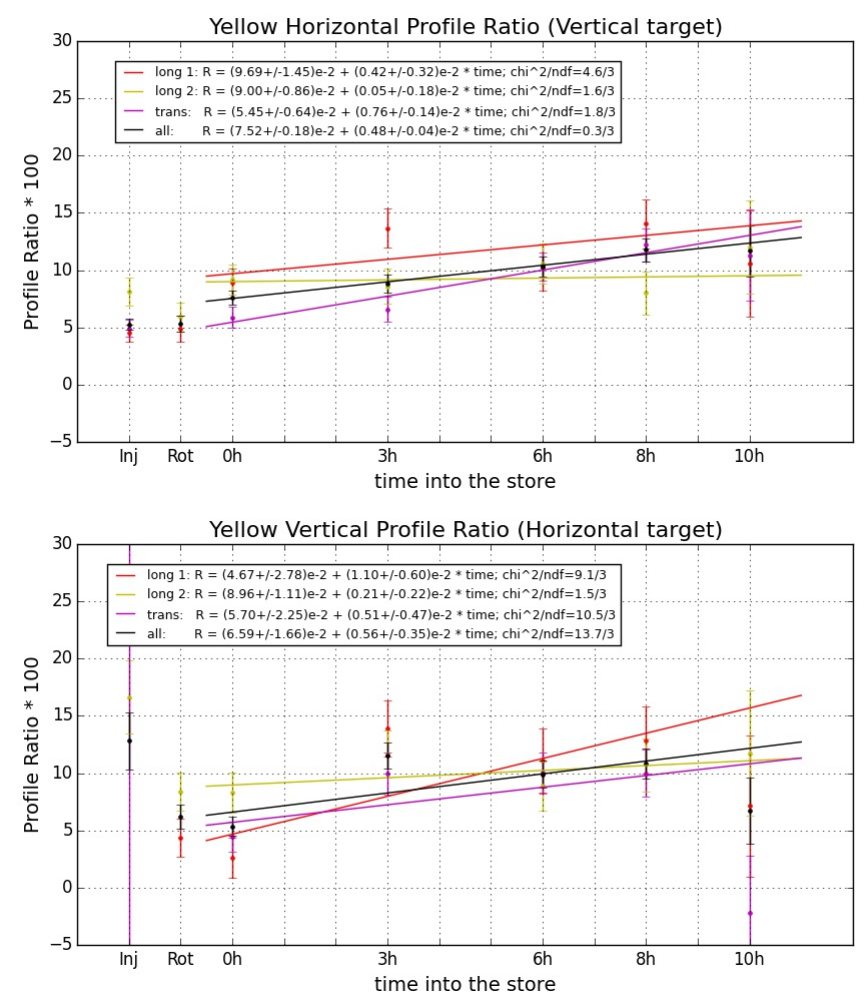

FIG. 6: Yellow profile ratio from vertical targets (top) and horizontal targets (bottom).

\begin{tabular}{|c|cccc|}
\hline Period & Blue Vertical & \multicolumn{3}{c|}{ Blue Horizontal } \\
\hline & Polarization & Decay Rate $(\% / \mathrm{hr})$ & Polarization & Decay Rate $(\% / \mathrm{hr})$ \\
\hline Long. I & $64.42 \pm 1.00$ & $0.94 \pm 0.20$ & $62.32 \pm 1.20$ & $0.84 \pm 0.23$ \\
\hline Trans & $61.35 \pm 0.55$ & $0.93 \pm 0.12$ & $59.95 \pm 0.57$ & $0.75 \pm 0.12$ \\
\hline Long.II & $56.47 \pm 0.71$ & $1.35 \pm 0.13$ & $56.05 \pm 0.88$ & $1.50 \pm 0.15$ \\
\hline All & $60.80 \pm 0.57$ & $1.21 \pm 0.11$ & $59.86 \pm 0.66$ & $1.17 \pm 0.13$ \\
\hline & Yellow Vertical & Yellow Horizontal \\
\hline Long.I & $65.80 \pm 0.89$ & $0.57 \pm 0.18$ & $65.13 \pm 1.10$ & $0.76 \pm 0.23$ \\
\hline Trans & $60.23 \pm 0.55$ & $0.51 \pm 0.11$ & $59.90 \pm 0.58$ & $0.60 \pm 0.12$ \\
\hline Long.II & $58.03 \pm 0.18$ & $0.49 \pm 0.03$ & $55.09 \pm 0.96$ & $0.40 \pm 0.18$ \\
\hline All & $61.07 \pm 0.51$ & $0.56 \pm 0.10$ & $59.66 \pm 0.83$ & $0.63 \pm 0.17$ \\
\hline
\end{tabular}

TABLE I: Fitted polarization at the beginning of store and polarization decay rate at store.

small spin mismatch between accelerators) and throughout the store.

Over the pp run, the source polarization is close to a constant level (as shown in Fig. 1). With the small spin mismatch at AGS injection due to partial snakes, the expected polarization at AGS injection is $80.2 \%$, which is the $P_{0}$ for AGS. The spin transfer efficiency at AGS injection with two partial snakes $(2.14 \mathrm{~T}$ for cold partial snake, $1.53 \mathrm{~T}$ for warm partial snake) is 0.996 [5] and RHIC injection spin transfer efficiency is 0.958 for blue and 0.991 for yellow, respectively [6]. With the source polarization as $82 \%$, the expected $P_{0}$ is $80.9 \%$ for yellow and $78.2 \%$ for blue, respectively.

Fig. 7 shows $P_{0}$ in blue ring at store. Both constant and linear fits were applied to see which one gives better fit. A better fit with significantly negative slope would indicate polarization loss due to imperfection resonances (no emittance dependence). As shown in Fig. 7, the slope is consistent with zero within error bars except for transverse case, which shows a small positive slope. This positive slope is not understood. Nevertheless, all the results are consistent with non-negative slope. This means that $P_{0}$ did not reduce its value over store. The difference in $P_{0}$ level among three periods can not be explained by AGS based on our polarization loss model, but more likely instrumental(see details later).

Fig. 8 shows $P_{0}$ in yellow ring at store with both constant and linear fits. Similar to blue case, there is no appreciable 

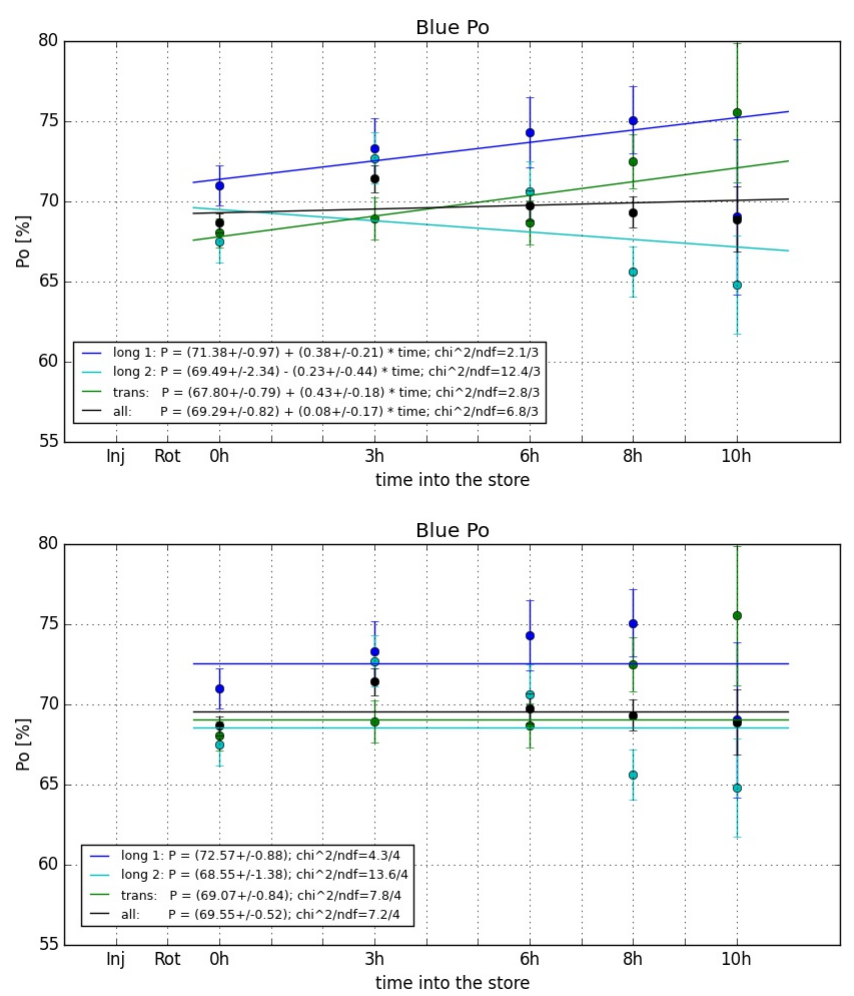

FIG. 7: Zero emittance polarization $P_{0}$ in blue ring at store: linear and constant fit. From theory, it should be a constant through store, if polarization loss is due to intrinsic resonances, associated with betatron motion amplitude. The linear fit is test if it can fit the data better or not.

\begin{tabular}{|c|cc|}
\hline Period & Blue & Yellow \\
\hline Long. I & $72.57 \pm 0.88$ & $76.02 \pm 1.51$ \\
\hline Trans & $69.07 \pm 0.84$ & $67.54 \pm 0.93$ \\
\hline Long.II & $68.55 \pm 1.38$ & $65.69 \pm 0.87$ \\
\hline All & $69.55 \pm 0.52$ & $69.11 \pm 0.36$ \\
\hline
\end{tabular}

TABLE II: Averaged zero emittance polarization $P_{0}$ at store.

negative slope in any of the three periods. Clearly, there is difference in $P_{0}$ among the three periods. Both blue and yellow ring results show that $P_{0}$ is lower over time as intensity was pushed higher.

The $P_{0}$ should be independent of intensity or which period. This is different from Table II. The difference among three periods suggests that the $R$ values derived from the polarimeter analysis are intensity dependent, or instrumental effect. The analysis should be revisited.

The polarization ratio between RHIC injection and AGS extraction are plotted in Fig. 9. There were several AGS extraction polarization measurements for each RHIC fill in the later part of the run. These values are averaged for each fill. The AGS extraction measurement is converted to averaged polarization of the whole beam based on the assumption that beam profile is the same in both transverse dimensions and the polarization loss is only due to intrinsic resonances. If the polarization profile is assumed round one, $R_{x}=R_{y}=R$, then the relation between fixed target measured polarization and zero-emittance polarization $P_{0}$ is

$$
P_{\text {peak }}=\frac{P_{0}}{(1+R)^{3 / 2}}
$$

On the other hand, the average polarization for a whole beam $P_{\text {ave }}$ is given by

$$
P_{\text {ave }}=\frac{P_{0}}{(1+R)^{2}}
$$



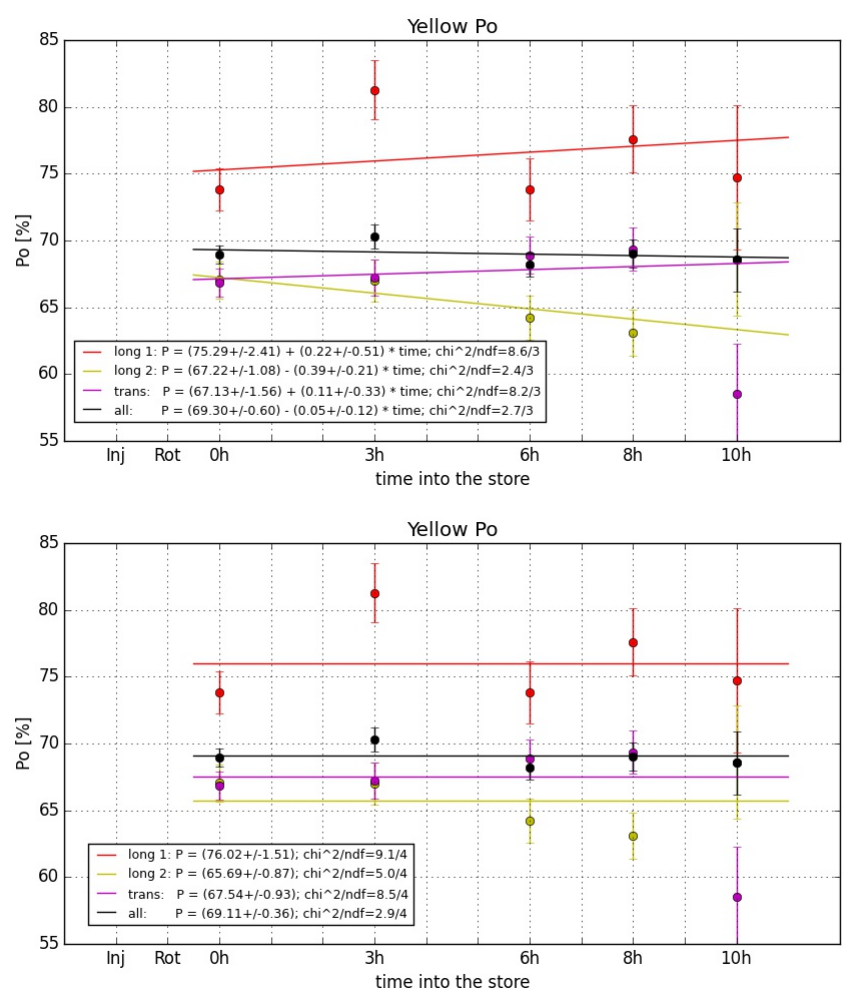

FIG. 8: Zero emittance polarization $P_{0}$ in blue ring at store: linear and constant fit.

Suppose we know $P_{0}$ (from averaged source polarization and spin projection at AGS injection), we can calculate the $R$ value at AGS extraction:

$$
(1+R)=\left(\frac{P_{0}}{P_{\text {peak }}}\right)^{2 / 3}
$$

Substitute it into Eq.(2), we have

$$
P_{\text {ave }}=P_{\text {peak }}\left(\frac{P_{\text {peak }}}{P_{0}}\right)^{1 / 3}
$$

This formula is used to derive the averaged polarization based on fixed target polarization measurement. One can get the averaged $R$ value for AGS extraction. The averaged AGS extraction polarization for all RHIC pp run fills during pp run is $68.5 \pm 0.3 \%$. From Eq.(4), $R=0.110 \pm 0.004$ for a round polarization profile. This is far from the values reported by RHIC polarimeters: $0.05-0.07$. The yellow polarization scaling factor 1.056 (average of yellow1 and yellow2) was applied for the yellow data. This scaling factor comes from store polarization calibration from polarized jet. Without applying it to injection, the polarization transmission efficiency on the ramp will be larger than one. Since polarization can only be lost but not increased on ramp, the factor is applied but it is the low limit for the scaling factor. Due to the spin mismatch at RHIC injection [6], we expect polarization is lower by a factor 0.958 for blue ring and a factor 0.991 for yellow ring.

Similarly, from Table I, one can get the $R$ values for blue and yellow rings respectively. The expected $P_{0}$ is $80.9 \%$ for yellow and $78.2 \%$ for blue, respectively. The calculated $R$ values at the beginning of store is 0.13 for blue and 0.15 (use vertical target results) for yellow for all pp run fills. These numbers are not too far from the value out of AGS. For the whole store, the averaged jet polarization is $53.0 \%$ for blue and $57.3 \%$ for yellow. The average $R$ values for stores can be estimated the same way: $R=0.21$ for blue and $R=0.19$ for yellow. From Figs. 5-6, the averaged $R$ values for all stores are 0.12 for blue and 0.10 for yellow, respectively. These are far below the $R$ values ( 0.2 ) derived from above model.

If there is no intensity dependent factor in the AtR spin transfer efficiency, the ratio of AGS average polarization to RHIC one is expected to be 1 and independent of intensity, after taking out the spin mismatch factor at RHIC injection. The ratios for each RHIC fills are plotted in Fig. 9 for both blue and yellow ring. Instead, the constant fitting of this ratio is about 0.92 for both blue and yellow. 

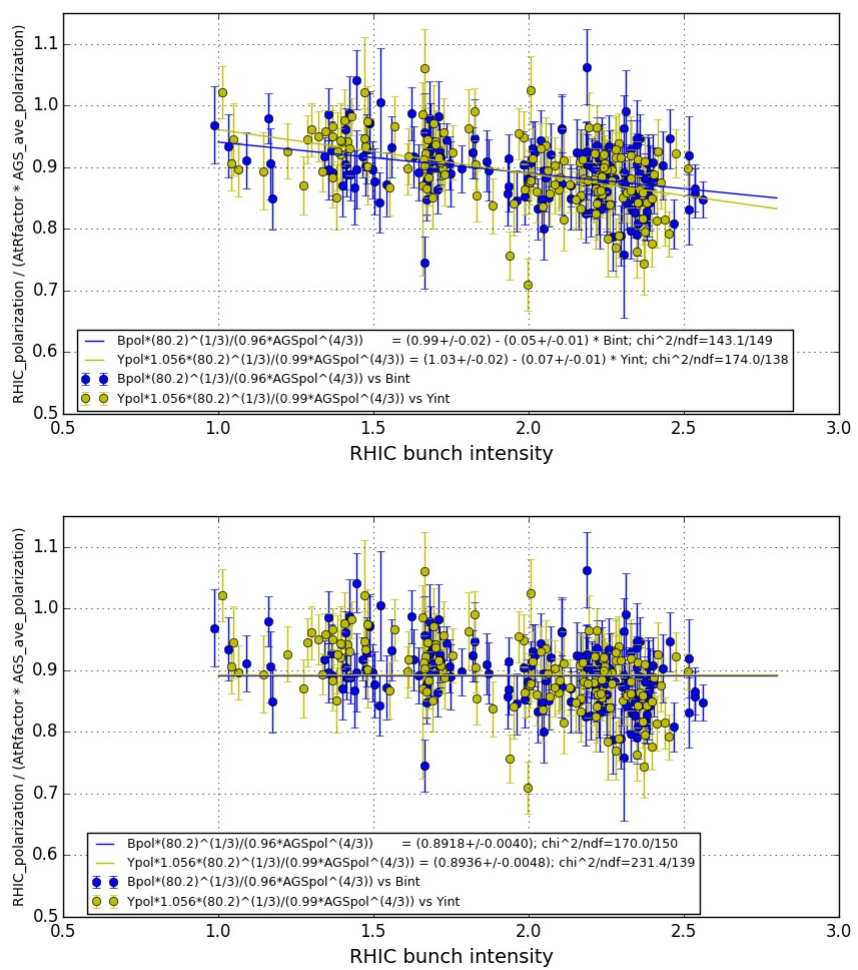

FIG. 9: Ratio of RHIC injection polarization and AGS average polarization. The AGS average polarization is converted from peak polarization measurement. The horizontal axis is the bunch intensity obtained from FDAview for blue ring and yellow ring, respectively.

First of all, it is good that both blue and yellow show the same factor, which indicates that there is no systematic difference between the blue polarimeters and yellow polarimeters. Second, the deviation from 1 needs some further analysis. Both AGS and RHIC polarimeters are CNI carbon polarimeter and use Si detectors. The analyzing power used in both polarimeters is derived from same experiment results. These have been under vigorous scrutiny in the past. As the instantaneous rate in AGS is very high, the AGS polarimeter analysis has a rate correction factor, which gives a correction of polarization in the order of $5 \%$ for fixed target measurement in 2-2.5E11 intensity range. It should be pointed out that with this correction introduced, the AGS extraction polarization $\left(P_{\text {peak }}, R_{x}, R_{y}\right.$ then $\left.P_{0}\right)$ are consistent with $P_{0}$ we assumed at AGS injection based on source polarization. So it seems there is a consistent picture on the AGS side. However, it shows a difference of $8 \%$ at RHIC injection, even with the analyzing power correction at store (with polarized jet) is applied. The next question is if we can look at the $R$ values to see if they provide similar difference. Unfortunately, there is problem with $R$ value measurements. The averaged $R$ values measured at RHIC injection is smaller than what measured at AGS extraction.

Third, the linear fit seems a little bit better than a constant fit. Although the chi square is only improved a little bit, the zero intensity ratio is very close to 1 . This leads to the question if the AtR spin transfer efficiency is sensitive to intensity. A question is if the particles with larger momentum spread associated with higher intensity may have different AtR spin transfer efficiency. Dr. Meot studied the spin transfer efficiency for different initial betatron motion amplitudes at the extraction point. The results show that the difference is relative small [7]. The next possibility is instrumentation (polarimeter) related. When the target goes through the beam, it is deformed by the electromagnetic forces as shown in Fig.10. Such deformation distorts the polarization profile and beam profile information. Consequently, the average polarization and $R$ value are also affected. However, such a effect would make both beam and polarization profiles flatter, so it is not straightforward that if the $R$ value would go up or down.

It is also possible that the overlapping resonances due to nearby imperfection resonance and intrinsic resonance may have different behavior which could have a polarization loss component independent of betatron motion, similar to imperfection resonance. This can be studied with the new model proposed by Dr. Ranjbar. [8]. 


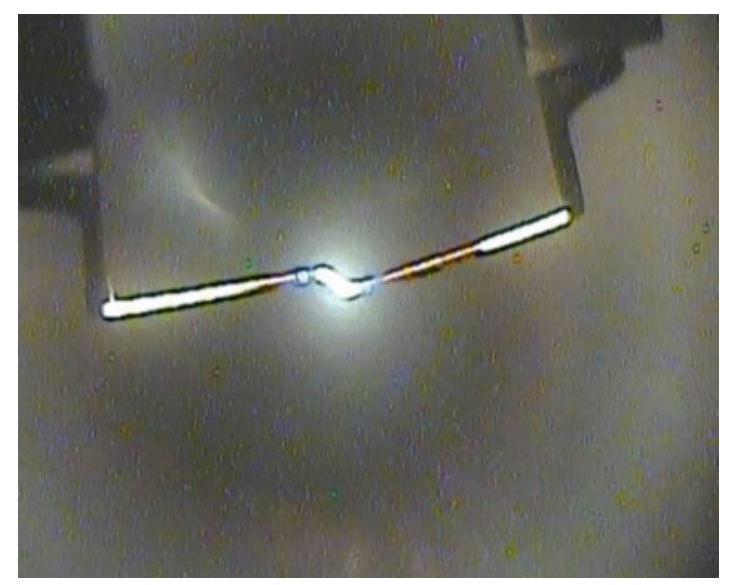

FIG. 10: The carbon target is twisted when crossing beam center. The measurement is at injection.

\begin{tabular}{|c|cccc|}
\hline Period & Injection & Fitted @Store & AGS Ext. & Intensity \\
\hline Blue & & & & \\
\hline Long. I & $64.85 \pm 0.30$ & $62.32 \pm 1.12$ & $72.41 \pm 0.40$ & ramping higher \\
\hline Trans & $59.86 \pm 0.25$ & $59.95 \pm 0.49$ & $68.73 \pm 0.29$ & ramping,leveled \\
\hline Long.II & $56.83 \pm 0.29$ & $56.05 \pm 0.77$ & $65.87 \pm 0.40$ & stay high \\
\hline Yellow & & & & \\
\hline Long.I & $68.42 \pm 0.26$ & $65.80 \pm 0.89$ & $72.41 \pm 0.40$ & ramping higher \\
\hline Trans & $60.94 \pm 0.23$ & $60.23 \pm 0.55$ & $68.73 \pm 0.29$ ramping,leveled \\
\hline Long.II & $59.29 \pm 0.35$ & $58.03 \pm 0.18$ & $65.87 \pm 0.40$ & stay high \\
\hline
\end{tabular}

TABLE III: Average polarization at AGS extraction, RHIC injection and beginning of store(fitted). Since only blue horizontal targets and yellow vertical targets were used at injection, only polarization measurements done by blue horizontal targets and yellow vertical targets from RHIC were included in the table. The general intensity is shown in 5th column.

\section{COMPARISON FOR INJECTION}

Table III shows the averaged polarization at AGS extraction, RHIC injection and beginning of RHIC store. The intensity was ramping higher through the whole Long.I period. It kept ramping higher and stopped in the middle of Trans. period. Part of the change of injection polarization is due to larger emittance associated with higher intensity. Part of it was due to no benefit with JQ on problem which started in the later part of transverse run, then come and goes through the rest of pp run. In general, polarization is trending lower in the AGS and both RHIC rings as intensity goes higher.

With the average polarization values, the polarization ratios can be calculated. They are given in Table IV. The expected ratio is calculated in the following way: 1. Assuming $80.2 \%$ polarization at AGS injection $\left(P_{0}\right)$, calculate

\begin{tabular}{|c|cccc|}
\hline Period & Expected $P_{i n j} / P_{A G S}$ & Measured $P_{\text {inj }} / P_{A G S}$ & $P_{\text {store }} / P_{\text {inj }}$ & $P_{\text {store }} / P_{A G S}$ \\
\hline Blue & & & & \\
\hline Long. I & $0.928 \pm 0.006$ & $0.896 \pm 0.006$ & $0.961 \pm 0.019$ & $0.861 \pm 0.017$ \\
\hline Trans & $0.912 \pm 0.006$ & $0.871 \pm 0.005$ & $1.002 \pm 0.010$ & $0.872 \pm 0.009$ \\
\hline Long.II & $0.899 \pm 0.007$ & $0.863 \pm 0.007$ & $0.986 \pm 0.016$ & $0.851 \pm 0.014$ \\
\hline All & $0.917 \pm 0.004$ & $0.870 \pm 0.004$ & $0.983 \pm 0.011$ & $0.855 \pm 0.010$ \\
\hline Yellow & & & & \\
\hline Long.I & $0.957 \pm 0.007$ & $0.945 \pm 0.006$ & $0.962 \pm 0.014$ & $0.909 \pm 0.013$ \\
\hline Trans & $0.940 \pm 0.005$ & $0.887 \pm 0.005$ & $0.988 \pm 0.010$ & $0.876 \pm 0.009$ \\
\hline Long.II & $0.927 \pm 0.008$ & $0.900 \pm 0.007$ & $0.979 \pm 0.007$ & $0.881 \pm 0.006$ \\
\hline All & $0.946 \pm 0.004$ & $0.903 \pm 0.004$ & $0.966 \pm 0.008$ & $0.873 \pm 0.008$ \\
\hline
\end{tabular}

TABLE IV: Ratio of polarization measured at AGS extraction $\left(P_{\text {peak }}\right)$ and RHIC $\left(P_{\text {ave }}\right)$. Only polarization measurements done by blue horizontal targets and yellow vertical targets from RHIC were included in the table. 


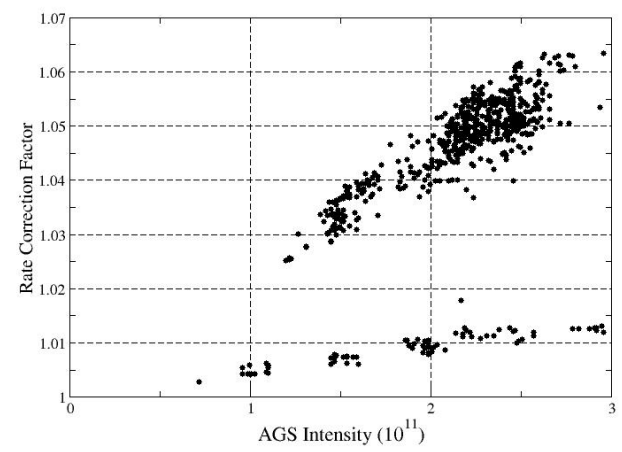

FIG. 11: The applied rate correction factors for one typical AGS vertical target (vertical 3) as function of bunch intensity. The apparent two bands are for fixed target measurements (the top band) and the sweep measurements (the bottom band). As can be seen, with lower rate in the sweep mode, the correction factor is almost not there.

the round polarization profile value $R\left(R_{x}=R_{y}, P_{\text {peak }}=P_{0} /(1+R)^{3 / 2}\right)$ and convert AGS number to whole beam, $P_{\text {ave }}=P_{\text {peak }} /(1+R)^{1 / 2}$. 2. Taking into account that injection miss match is 0.958 for blue ring and 0.991 for yellow ring. So the expected ratio is not going to be one for two reasons: the spin mismatch at RHIC injection and the difference between sweep measurement $\left(P_{\text {ave }}\right)$ and fixed target measurement $\left(P_{\text {peak }}\right)$. The error bar of expected ratio comes from the AGS extraction polarization measurements.

The expected ratio is getting lower for the three periods because the intensity (and emittance) are getting higher for the three periods. With larger polarization profile associated with higher intensity, the difference between RHIC (sweep measurement) and AGS (fixed target measurement) is getting larger. This is expected. The measured ratios are lower than the expected values but follow the intensity trend except in yellow for the transverse period.

The ratio of $P_{\text {store }} / P_{i n j}$ is particularly interesting because it comes from the same polarimeter and some systematic errors are canceled out. One would expect that this ratio is similar for the two longitudinal periods as we expect the two snakes should have no problem to maintain polarization on the ramp. The fact that both blue and yellow had higher ratio for longitudinal period 2 suggests it could be instrumental, namely, the intensity effect on the polarimeter. The ratio $P_{\text {store }} / P_{i n j}$ is larger for transverse period, it may indicate that there is additional polarization loss in the extra rotator ramp. The ratio $P_{\text {store }} / P_{A G S}$ is similar for all three periods in two individual rings. It also shows that the ratio is better for yellow ring reflecting the fact that the yellow ring spin transmission efficiency is about $3 \%$ better than blue. This implies that the difference of the three periods are mainly BEFORE RHIC injection measurements: it is due to AGS(likely) or AtR (no known mechanism). Nevertheless, there are polarization loss as reflected by the polarization decay rate. There are more loss in blue at store, especially the 3rd period. Within error bars, polarization ratio of $P_{\text {store }} / P_{i n j}$ is lower and similar for both blue (horizontal target) and yellow (vertical target).

The expected ratio $P_{i n j} / P_{A G S}$ goes down as intensity was ramped through the run. This is due to the larger polarization profiles associated with larger emittance for higher intensity. For blue, the measured ratio is about 3-5\% lower than the expected ratio. There is no noticeable difference among the three periods. For yellow the variation in the difference is larger, the measured ratio is about 1-6\% lower than the expected ratio. Since there is rate correction in AGS polarimeter, the rate correction dependence on intensity is also explored. Fig. 11 shows the correction factor as function of bunch intensity for a typical vertical target (V3).

The majority of the pp run intensity in the three periods were between 1.0E11 to2.5E11 bunch intensity. From Fig. 11, the correction factor varies between 1.02 to 1.06. This correction factor is in the order of the difference between AGS extraction and RHIC injection. If the rate correction is removed, the agreement between AGS and RHIC will be closer. However, the instantaneous rate of AGS polarimeter is about 7-8 times higher than RHIC due to the target size difference. It is known that we have rate problem and a rate correction is needed. Second, the derived $P_{0}$ is consistent with the input polarization with this rate correction. In short, the expected ratio shows that the AGS polarization measurement should be about 5-10\% higher in AGS with the current intensity and measurement types. The measured ratios are a few percents lower than that. 


\section{SUMMARY}

The analyses confirm the consistency among polarization values and polarization decay between two polarimeters in each ring. The jet calibration only gives the right values for the overall polarization. The polarization decay rates from polarimeters 1 and 2 are consistent is not a given. However, there are larger error in $R$ values, and this may resulted non-consistent zero emittance polarization value $P_{0}$ between AGS and RHIC. Here are the answers to the three questions posted in the introduction.

1. The big difference between polarization at AGS extraction and expected RHIC injection( $4-8 \%$ for yellow and blue rings, respectively) is expected due to difference between fixed target measurement and sweep measurement. The percentage of polarization ratio referred in this paper is relative difference. In future operation, one should not be surprised by the $4-8 \%$ difference. The difference is expected to be larger when beam intensity is higher and consequently emittance is larger. The expected ratio (RHIC over AGS) is about 3-4\% higher than the measured ones, but the difference in different periods follows the expected trend for both blue and yellow. This implies that AGS average polarization $P_{\text {ave }}$ (not $P_{\text {peak }}$ ) is $2-3 \%$ (absolute value) higher than what RHIC injection values after all the corrections are put in. There is no plausible explanation for this. One key fact is that we have no jet calibration at RHIC injection with small enough error bar to define the analyzing power at injection. Another jet measurement at RHIC injection with small enough error bar (2-3\% statistically) would be needed. The additional complication here is that the $R$ values at RHIC injection are not consistent with AGS ones. The ratio of RHIC injection polarization and AGS average polarization shows intensity dependence (Fig. 9). This is a puzzle.

2. There is not much difference in yellow polarization decay (slope of the polarization loss) among the three different periods. The difference of the polarization in the three periods is due to the AGS polarization difference. These analyses confirm that the polarization decay slopes are similar but initial polarization values are different. The polarization difference at injection and beginning of store among the three periods are correlated with the difference of AGS polarization for the three periods. This implies that the difference of polarization in yellow ring over the three periods comes from AGS. There is no additional polarization loss at store due to higher bunch intensity. Usually, larger emittance comes with higher bunch intensity. Either the emittance is not larger for the higher bunch intensity, or the emittance impact is not large enough to be visible( which implies that the two snakes can handle this emittance increase up to $100 \mathrm{GeV}$ ). In general, the polarization loss on the ramp is about $2-3 \%$, mainly in the rotator ramp part. It should be noted that this loss is based on the assumption that the analyzing power at injection is the same as at $100 \mathrm{GeV}$, which we have no firm experiment results to prove that. The loss rate in store is about $0.6 \% /$ hour. There is an unknown source difference between RHIC injection and AGS extraction, the yellow injection is about 2-3\% lower (as stated in above paragraph). For blue, the larger polarization decay rate relative to yellow indicates blue lattice is worse than yellow. The 3rd period (longitudinal II) has much stronger polarization decay slope. The larger blue polarization decay rate could be due to difference in optics and possibly e-lens from yellow. Some modeling of the real machine is needed to explain the difference.

3. The polarization with zero emittance $P_{0}$ is close to a constant through stores as expected. But its level is lower than what AGS reported and changed for the three periods, likely due to intensity change. The averaged $P_{0}$ over the whole pp run is shy of $70 \%$ for both blue and yellow rings, but the expected value should be around $80 \%$. This may imply that the $R$ value is not properly calculated. One obvious reason (complication) would be the target deformation during polarization measurement. The analysis process probably should be revisited. The offline analysis may provide a few percents higher jet polarization [9], but it would not change the derived $R$ values significantly.

Last but not the least, the ratio of polarization transmission efficiency in RHIC suggests that the polarization loss during rotator ramp is real: the efficiency of transverse period is better in both blue and yellow. Considering the length of rotator ramp, the polarization loss rate here is comparable to energy ramp (if we believe the analyzing power at injection) and much stronger than at store. Since this is a relative shorter time than store, the spin tracking study of this period could be easier.

\section{Acknowledgment}

The authors would like to thank L. Ahrens, E. Aschenauer, Y. Makdisi, F. Meot, N. Tsoupas, V. Ranjbar, V. Schoefer and A. Zelenski for fruitful discussion. The authors would also like to thank O. Eyser, A. Poblaguev, B. Schmidke and G. Webb for offline polarization data. Lastly, the authors would like to thank V. Schoefer and F. Meot 
on the help of emittance data and analysis of spin direction at AtR.

[1] H. Huang, et al., in Proc. of IBIC14, p.124.

[2] E. Aschenauer, in RHIC retreat 2015.

[3] http://www.phy.bnl.gov/cnipol/rundb/

[4] W. Fischer and A. Bazilevsky, Phys. Rev. STAB 15. 041001(2012).

[5] F. Meot, Spin Meeting Presentation Jan. 30, 2013.

[6] W.W. Mackay, N. Tsoupas, in Proc. of EPAC2006, p.1795.

[7] F. Meot, Private communication.

[8] V. Ranjbar, Phys. Rev. STAB 18. 014001(2015).

[9] A. Poblaguev, Private communication. 